\title{
Concomitant use of
}

\section{ibuprofen and paracetamol}

\section{and the risk of major clinical}

\section{safety outcomes}

\section{Frank de Vries, ${ }^{1,2,3}$ Efrosini Setakis ${ }^{1} \&$ Tjeerd-Pieter van Staa ${ }^{1,2}$}

${ }^{1}$ General Practice Research Database, Medicines and Healthcare products Regulatory Agency (MHRA), London, UK, ${ }^{2}$ Utrecht Institute for Pharmaceutical Sciences, Utrecht University, Utrecht, the Netherlands and ${ }^{3}$ MRC Epidemiology Resource Centre, Southampton General Hospital, Southampton, UK

\section{Correspondence}

Dr Tjeerd-Pieter van Staa, General Practice Research Database, Medicines and Healthcare products Regulatory Agency, 1 Nine Elms Lane, London SW8 5NQ, UK. Tel.: + 442070842019

Fax: + 442070842041

E-mail:Tjeerd.vanstaa@GPRD.com

\section{Keywords}

paracetamol, anti-inflammatory agents, drug toxicity, ibuprofen, mortality, non-steroidal

Received

5 February 2010

Accepted

11 April 2010

\section{WHAT IS ALREADY KNOWN ABOUT} THIS SUBJECT

Non-steroidal anti-inflammatory drugs (NSAIDs) and paracetamol are widely used analgesics in the prescription and non-prescription settings. Although both classes of drug are generally well tolerated, they can lead to well-characterized adverse effects. Both drugs are widely co-prescribed and it is of interest to understand better safety outcomes when the two drugs are taken concomitantly.

\section{WHAT THIS STUDY ADDS?}

Relative rates and hazard ratio patterns of safety outcomes were broadly similar for patients prescribed ibuprofen alone, paracetamol alone and concomitant ibuprofen and paracetamol. The risks of the various safety outcomes examined do not appear to be modified by concomitant use of ibuprofen and paracetamol compared with paracetamol or ibuprofen alone.

\section{AIMS}

To evaluate and compare the risk of specific safety outcomes in patients prescribed ibuprofen and paracetamol concomitantly with those in patients prescribed ibuprofen or paracetamol alone. The outcomes were evaluated according to dose, duration and exposure.

\section{METHODS}

The study used a retrospective longitudinal cohort design with data from the UK General Practice Research Database (GPRD). The study population included patients aged 18 years or over who were prescribed ibuprofen alone, paracetamol alone or concomitant ibuprofen and paracetamol (tablets or capsules only). The safety outcomes evaluated were upper gastrointestinal events, myocardial infarction, stroke, renal failure (excluding chronic), congestive heart failure, intentional or accidental overdose, suicidal behaviour and mortality. Time-dependent Cox regression was used to estimate relative rates for the safety outcomes, by treatment group. A further analysis evaluated whether the hazard rates (i.e. absolute risks) varied over time with changes in drug exposure.

\section{RESULTS}

The study population included 1.2 million patients. There was considerable heterogeneity in both patient and exposure characteristics. When comparing with past users, for most safety outcomes, current users of concomitant paracetamol and ibuprofen had relative rates between those for current users of ibuprofen alone and paracetamol alone. The hazard rates were generally proportional over time, from current to past exposure, following a prescription for concomitant paracetamol and ibuprofen compared with ibuprofen alone or paracetamol alone.

\section{CONCLUSIONS}

The known risk of the safety outcomes examined does not appear to be modified by concomitant use of ibuprofen and paracetamol compared with paracetamol or ibuprofen alone. 


\section{Introduction}

Ibuprofen and paracetamol are widely used analgesics. Although both drugs are readily available as over the counter (OTC) medications, they are also available on prescription. Although nonsteroidal anti-inflammatory drugs (NSAIDs) such as ibuprofen are generally well tolerated, infrequent but potential adverse effects include upper gastrointestinal (Gl) bleeding and perforation, renal failure and heart failure [1]. The objective of this retrospective cohort study was to evaluate a range of safety outcomes in patients prescribed ibuprofen and paracetamol concomitantly and compare these with safety outcomes in patients prescribed ibuprofen or paracetamol alone. Specifically, these outcomes were assessed with reference to the dosage and treatment duration.

\section{Methods}

\section{Data source}

The study data was sourced from the UK General Practice Research Database (GPRD). The GPRD contains anonymized computerized medical records from general practitioners (GPs). The records include demographic information, prescription details, clinical events, provision of preventive care, details of specialist referrals and hospital admissions and major outcomes [2, 3]. The GPRD data collection started in 1987 and currently includes data on approximately 10 million patients (http://www.gprd.com/ home).

\section{Study population}

The study cohort included patients aged 18 years or older who received a prescription for ibuprofen or paracetamol (tablets or capsules only) between 1987 and August 2007. The date of the first prescription of ibuprofen or paracetamol during this data collection period was defined as the index date. The follow-up period was from the index date to August 2007 or the date the patient transferred out of the practice, or the date of death, whichever was earliest. The study was reviewed scientifically by the Independent Scientific Advisory Committee of GPRD and approval by the Trent ethics committee was given for research with anonymized GPRD data.

\section{Safety outcomes}

Safety outcomes were assessed with OXMIS and Read codes, and included: mortality, upper Gl events (gastroduodenal ulcers and complications such as upper $\mathrm{Gl}$ haemorrhage), myocardial infarction (MI), stroke, acute renal failure, congestive heart failure, overdose (intentional or accidental) and suicidal behaviour. Suicidal behaviour included self-laceration, overdose (irrespective of the type of chemical) or suicidal thoughts. These medical terms are based on those used by Martinez and colleagues [4] in another GPRD study.

\section{Exposure definitions}

The total period of follow-up was divided into periods of 'current', 'recent' and 'past' exposure using the following definitions: current exposure, the period from date of the prescription to 3 months after the estimated end of the prescription; recent exposure, the period 3-6 months after the estimated end of the prescription; past exposure, the period $\geq 6$ months after the estimated end of the prescription. Each exposure period was then classified into'ibuprofen only,' 'paracetamol only' and 'concomitant paracetamol and ibuprofen' using the following definitions: (i) ibuprofen only, with no prescriptions for paracetamol, other NSAIDs and aspirin in the preceding 6 months, (ii) paracetamol only, with no prescriptions for ibuprofen, other NSAIDs and aspirin in the preceding 6 months and (iii) concomitant paracetamol and ibuprofen prescribed on the same date, with no prescriptions for other NSAIDs and aspirin in the preceding 6 months. As exposure may vary over time, patients were classified in a time-dependent manner with patients moving between exposure categories over time.

In order to evaluate the association between outcomes and frequency of exposure, current users were classified into seven groups based on the exposure characteristics: (i) first prescription (Rx), patients who received their first ibuprofen or paracetamol prescription at least 12 months after the start of GPRD data collection and who had not previously been prescribed aspirin or other NSAIDs, (ii) long gap, patients with at least 6 months between a preceding prescription for ibuprofen, paracetamol, aspirin or other NSAID and the current prescription for ibuprofen, paracetamol or concomitant ibuprofen and paracetamol, (iii) repeat use with a low medication possession ratio (MPR), for patients who had been prescribed ibuprofen and/or paracetamol in the preceding 6 months. The MPR is defined as the ratio of duration of the previous prescription, to the time between that prescription and the current prescription (equal to $<0.40$ ), (iv) repeat use with a medium MPR, as above but with ratio equal to $0.40-0.59$, (v) repeat use with a high MPR, as above but with ratio equal to $0.60-0.79$, (vi) repeat use with a very high MPR, as above but with ratio equal to $>0.8$ and (vii) repeat use with no information on the number of days prescribed, and consequently no information on compliance.'

\section{Statistical analyses - relative rates of safety outcomes}

Poisson regression models were used to estimate the relative rates (RRs and $95 \%$ confidence intervals) of the safety outcomes in current users of ibuprofen alone, paracetamol alone or concomitant ibuprofen and paracetamol. The RRs were adjusted for age, gender, calendar year, body mass index, smoking history, alcohol use, number of visits to the GP in the previous 6-12 months, hospital admission in the 
previous year and socioeconomic status in the location of the practice. Prescribing of other types of NSAIDs or aspirin in the preceding 6 months was also noted. Additional risk factors in the statistical adjustment specific for each of the safety outcomes included: (i) for mortality, the additional risk factors were a history of: upper Gl events, osteoarthritis, rheumatoid arthritis, ischaemic heart disease, heart failure, hypertension, cerebrovascular disease, diabetes mellitus, hyperthyroidism, stroke or transient ischaemic attack, cancer (excluding non-melanoma skin cancer), inflammatory bowel disease, autoimmune disease (systemic lupus erythematosus, systemic sclerosis, vasculitis, rheumatoid arthritis), depression, drug abuse and prescribing in the previous 6 months (anticoagulants, oral glucocorticoids, diuretics, cardiac glycosides, statins, angiotensin receptor blockers, hypnotics and anxiolytics, antipsychotic drugs, antibacterial drugs, aminosalicylates, antidepressants), (ii) for upper Gl events, the additional risk factors were a history of upper $\mathrm{Gl}$ events, osteoarthritis or rheumatoid arthritis, prior prescribing of anticoagulants, aspirin, oral corticosteroids, proton pump inhibitors, $\mathrm{H}_{2}$-receptor antagonists, (iii) for $\mathrm{Ml}$, the additional risk factors were a history of ischaemic heart disease, heart failure, hypertension, cerebrovascular disease, diabetes mellitus, hyperthyroidism, hyperlipidaemia, prior prescribing of diuretics, cardiac glycosides, statins, angiotensin receptor blockers, oral glucocorticoids, (iv) for stroke, the additional risk factors were a medical history of stroke or transient ischaemic attack, heart failure, hypertension, cerebrovascular disease, diabetes mellitus, hyperthyroidism, hyperlipidaemia, prior prescribing of diuretics, cardiac glycosides, statins, angiotensin receptor blockers, anticoagulants, oral glucocorticoids, (v) for heart failure, the additional risk factors were a history of ischaemic heart disease, hypertension, cerebrovascular disease, diabetes mellitus, hyperthyroidism, prior prescribing of diuretics, cardiac glycosides, statins, angiotensin receptor blockers, oral glucocorticoids, (vi) for renal failure, the additional risk factors were a history of cancer (excluding non-melanoma skin cancer), congestive heart failure, inflammatory bowel disease, autoimmune disease (systemic lupus erythematosus, systemic sclerosis, vasculitis, rheumatoid arthritis), diabetes mellitus, hypertension, prior prescribing of hypnotics and anxiolytics, antipsychotics, antibacterials, aminosalicylates, oral glucocorticoids and (vii) for overdose or suicidal behaviour, the additional risk factors were a history of depression, drug abuse, prior prescribing of antidepressants, antipsychotics.

\section{Pattern of risk over time following a prescription}

Hazard rates (i.e. absolute risk) were estimated over time following a prescription. The follow-up period was from the date of the prescription until the date of the next prescription or date of censoring, whichever was earliest. The total follow-up period was divided into 100 sub-periods and the absolute risk was estimated within each subperiod. These estimates were then smoothed using the methods proposed by Ramlau-Hansen [5]. For computational reasons, the hazard rates were estimated for a maximum of 100000 prescriptions and random subsamples were used for larger numbers. This analysis of hazard rates can be used to display visually the observed (crude) risks over time. The time close to a prescription is likely to include the greatest number of patients exposed to the drug, while the distant time is likely to include more patients who discontinued the drug. In traditional epidemiological studies, the time close to a prescription would be classified as current exposure and distant time as past exposure. Changes in rates over time (i.e. testing whether rates remained parallel over time or diverged/converged) were evaluated using the test for proportionality in Cox proportional hazards regression. Age, sex and calendar year at the time of the prescription were included in the regression analysis. This method has previously been used to study hazard rates of $\mathrm{Ml}$ and mortality in users of $\beta_{2}$-adrenoceptor agonists $[6,7]$.

\section{Results}

The study population included 1.2 million patients. Of these, 1.0 million had not been prescribed other NSAIDs or aspirin in the preceding 6 months. At the index date, mean ages were 47.5 years in the ibuprofen group, 62.5 years in the paracetamol group and 52.5 years in the concomitant ibuprofen and paracetamol group (Table 1). Patients in the paracetamol alone group were more likely to be on concomitant medication or have history of disease.

From the index date onwards, Table 2 shows that the patient population and the frequency of prescribing ibuprofen and/or paracetamol were different between groups. Ibuprofen alone was prescribed to a younger population (mean age 57.0 years) and less frequently than paracetamol alone (mean age 71.6 years) or concomitant ibuprofen and paracetamol (mean age 64.6 years).

As shown in Table 3, current users with continuous use (very high MPR) of ibuprofen (e.g. RR stroke 1.23, 95\% Cl $1.12,1.35$ ) or paracetamol (e.g. RR stroke $1.30,95 \% \mathrm{Cl} 1.19$, 1.41) generally had higher RRs, and those with intermittent drug use (low MPR) had lower RRs (e.g. RR stroke in ibuprofen users $0.99,95 \% \mathrm{Cl} 0.86,1.13$; RR stroke in paracetamol users $1.03,95 \% \mathrm{Cl} 0.97,1.10)$ compared with past users. The RRs of most outcomes were statistically similar in current users of ibuprofen alone, paracetamol alone and concomitant ibuprofen and paracetamol (based on the tests for interaction between the RRs). The RRs for the safety outcomes were statistically proportional over time, from current to past exposure, between the various medication classes, with the exception of renal failure (based on the tests for proportionality of RR over time). Figure 1 gives the crude hazard rates for each safety outcome following a 


\section{Table 1}

Characteristics of study population at baseline (index date)

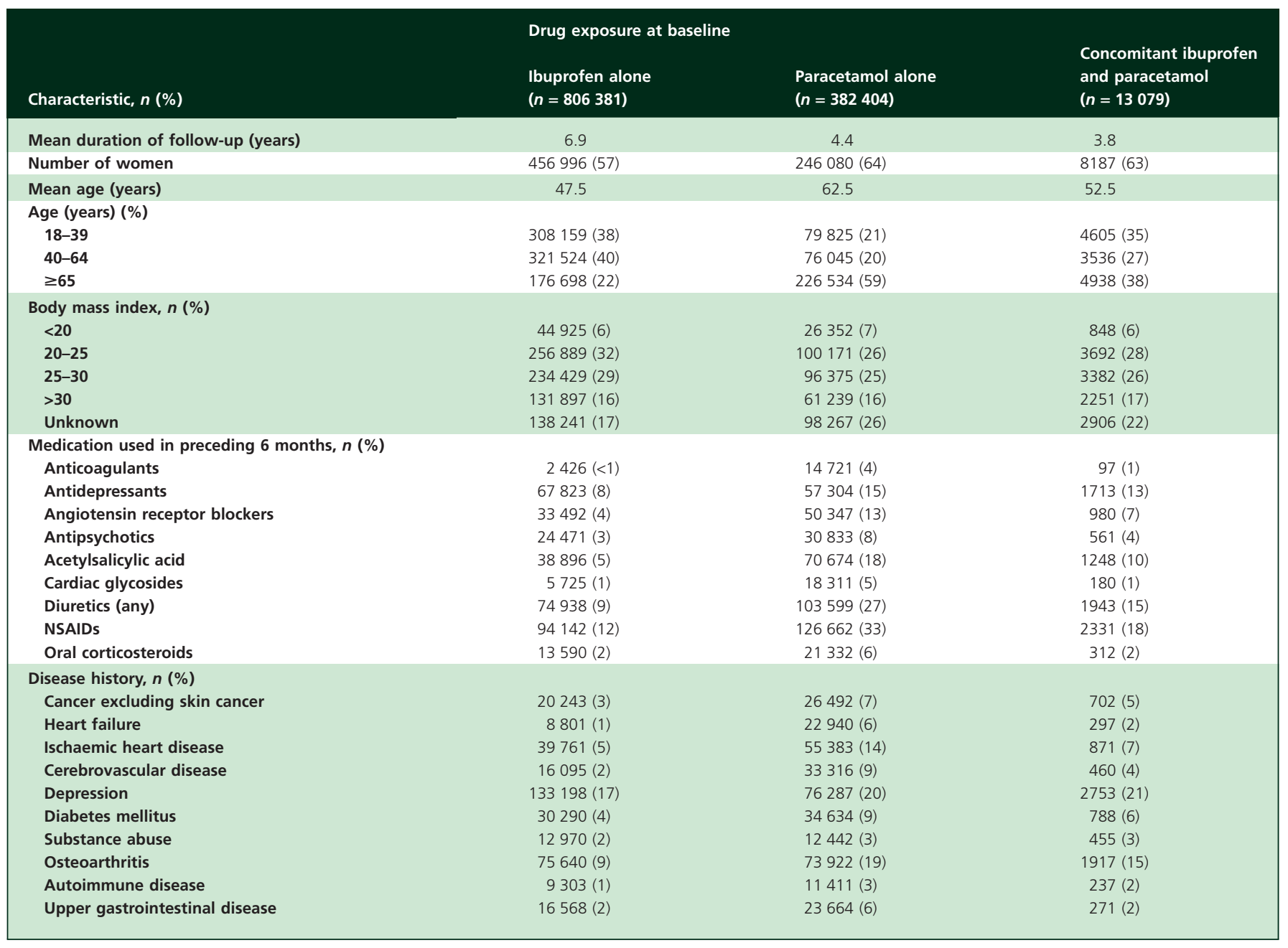

Percentages may not add up to $100 \%$ due to rounding. NSAIDs, non-steroidal anti-inflammatory drugs.

prescription, which were used in the statistical proportionality analysis.

\section{Discussion}

This study used data from the GPRD to evaluate the safety of concomitantly prescribed ibuprofen and paracetamol, ibuprofen alone and paracetamol alone. There was considerable between-group heterogeneity in the patient and exposure characteristics. An analysis of patterns of risks for safety outcomes over time and changes in exposure was conducted partly to overcome the issues of unmeasured confounding and bias in the study population.

\section{Mortality}

The RR of mortality had a U-shaped pattern in all medication classes, with a larger excess in patients without extensive prior use of ibuprofen or paracetamol and in patients with long-term continuous use of the same medication class. The most likely explanation for the higher risk in patients without extensive prior use is that these drugs were prescribed to patients with severe disease at increased risk of death. Patients with pain symptoms due to an exacerbation of a severe disease may have been more likely to visit the GP and be prescribed an analgesic. Large studies conducted in Denmark found similar confounding by indication with ibuprofen and, particularly, paracetamol $[8,9]$. The pattern of mortality risk over time showed that concomitant use of ibuprofen and paracetamol was statistically comparable with that of ibuprofen and paracetamol alone. The differences between these groups were of similar magnitude during current and past exposure, which does not support the presence of differential effects on mortality of these analgesics. 


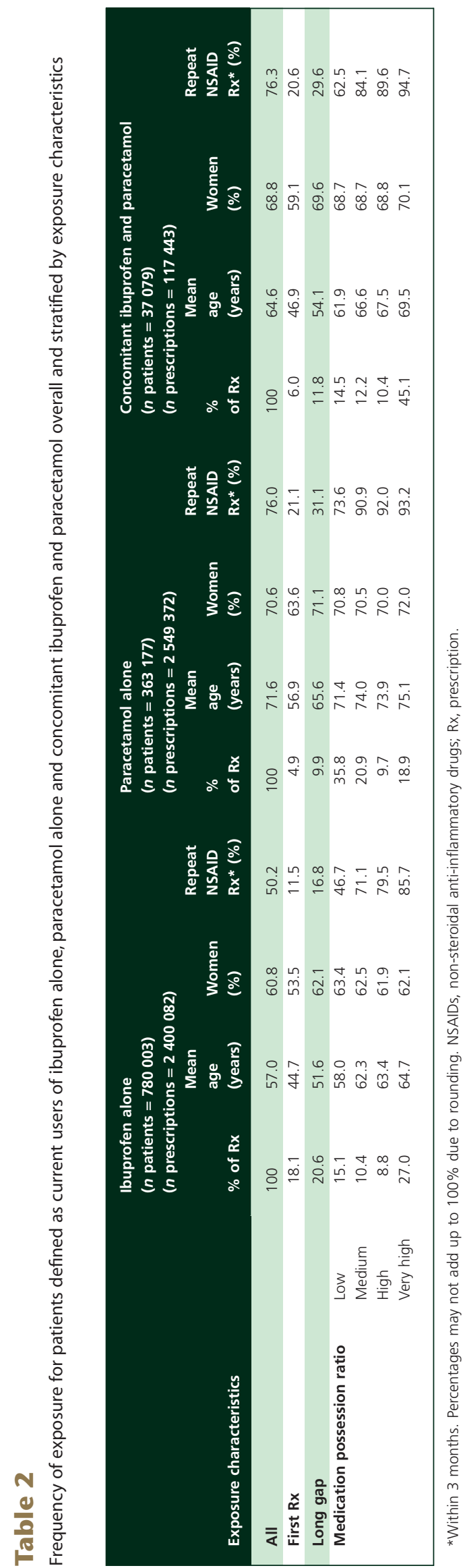

\section{Upper Gl events}

NSAIDs are known to cause upper Gl events [1]. In this study, observed RRs $(1.18,95 \% \mathrm{Cl} 1.13,1.24)$ tended to be lower than those reported previously $[10,11]$. A UK study conducted using data from another GP database reported an odds ratio of $1.42(95 \% \mathrm{Cl} 1.27,1.59)$ for ibuprofen [11], while an older study reported a RR for ibuprofen of 2.5 $(95 \% \mathrm{Cl} 1.9,3.4)[10]$. The differences between recent and older estimates for the $\mathrm{Gl}$ effects of ibuprofen may reflect the increased concomitant use of acid suppressants and/or the substantial reduction over calendar time in the rate of upper $\mathrm{Gl}$ events [12].

A Canadian study including over 640000 patients showed that the combination of a standard NSAID and paracetamol was associated with an increased risk of hospitalization for upper Gl events [13]. Patients prescribed paracetamol alone were more likely to be older and to have other concomitant disease compared with those prescribed standard NSAIDs alone [14].

Various characteristics of NSAID exposure were measured in our study. Although the GPRD does not contain data on the actual use of medications by patients, a low medication possession ratio may indicate intermittent use (i.e. insufficient medication for continuous use). We found that the rate of upper $\mathrm{Gl}$ events was higher in those patients with frequent NSAID use. A US case-control study in which patients were interviewed about their medication use found no increased risk of upper Gl events with infrequent NSAID use (either OTC or prescription), while frequent use was associated with a doubling of risk [15]. These findings indicate that comparisons between different analgesics should take into account exposure characteristics.

\section{Cardiovascular events}

Use of paracetamol at high dose or frequency has been associated with an increased risk of cardiovascular events [16]. Adverse cardiovascular effects have also been reported in randomized trials for selective COX-2 inhibitors [16-19]. However, patients included in randomized trials for selective COX-2 inhibitors are very different from patients in daily practice with respect to indications for analgesic use, daily dose and duration of use. The daily dose in patients using selective COX-2 inhibitors was twoto three-fold lower in the GPRD than in major RCTs [12]. Since 2004, there has been growing concern that some of the older NSAIDs, such as diclofenac, may have adverse cardiovascular effects similar to those of selective COX-2 inhibitors [20]. However, there are several challenges in establishing the causal contribution of NSAIDs to cardiovascular outcomes in daily clinical practice. Furthermore, a recent GPRD study found that patients who stopped NSAIDs after a long duration of use were at increased risk of MI [21].

In this study, we found no differences in the rate of MI between the three groups. However, the risk of MI was 


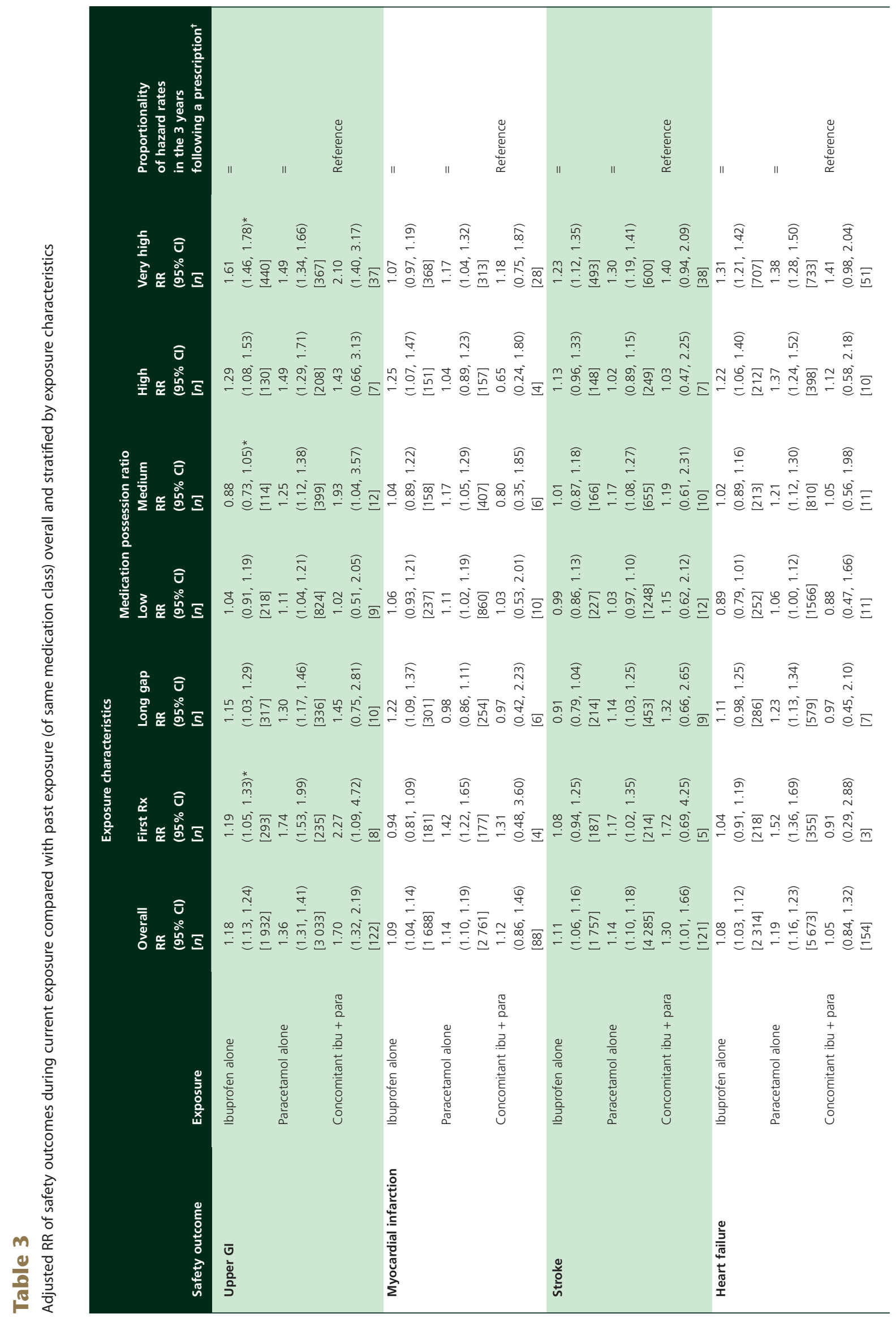




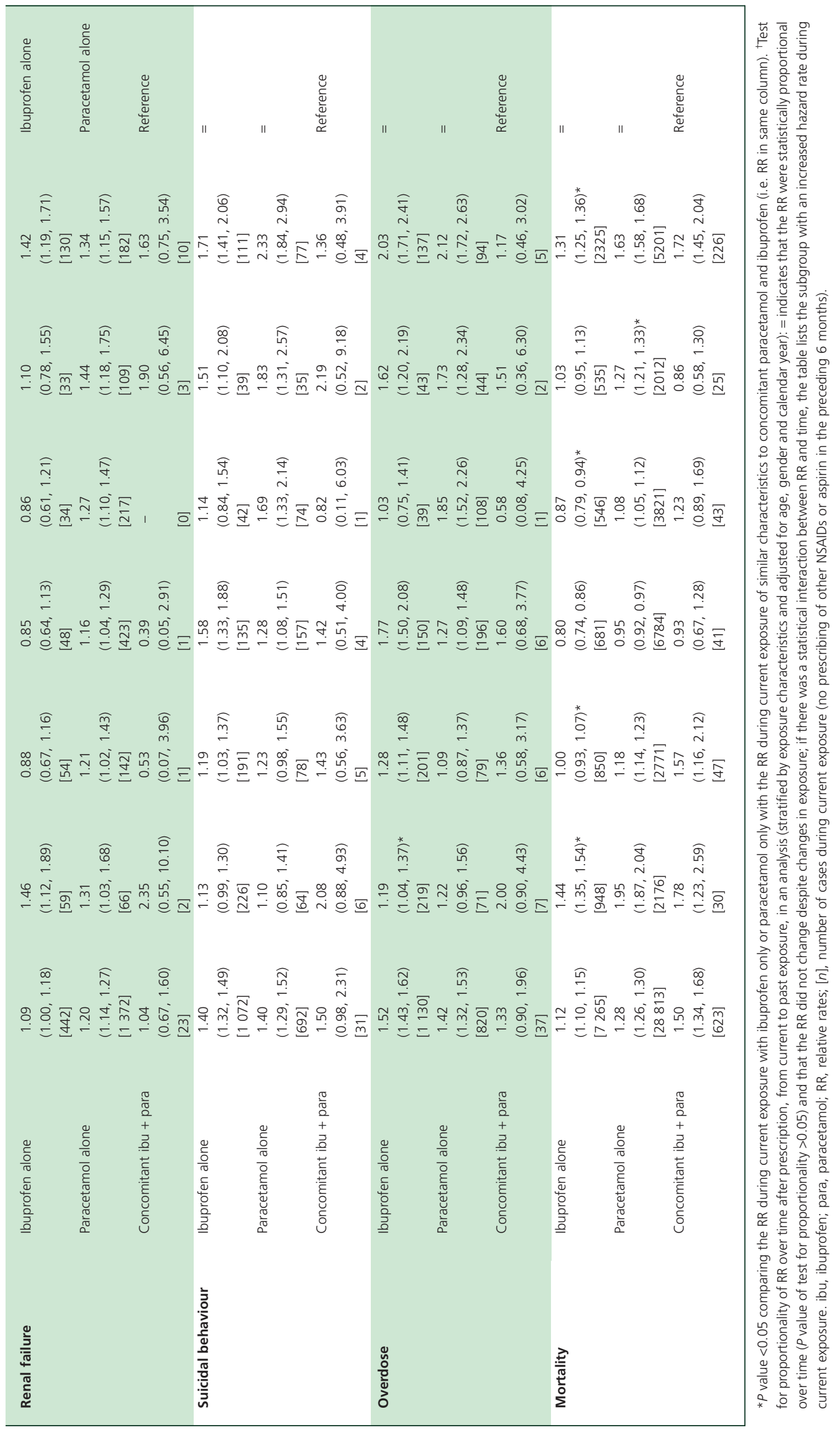


Upper gastrointestinal events

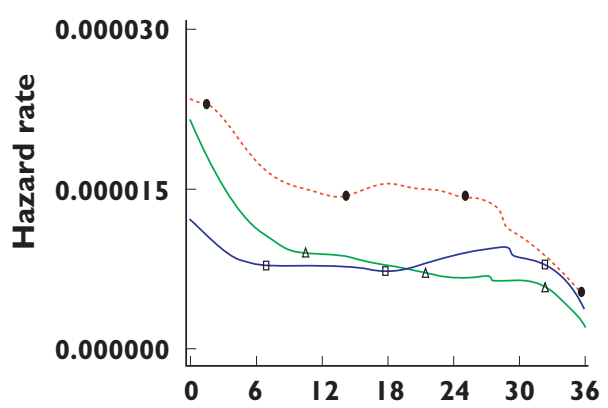

Heart failure

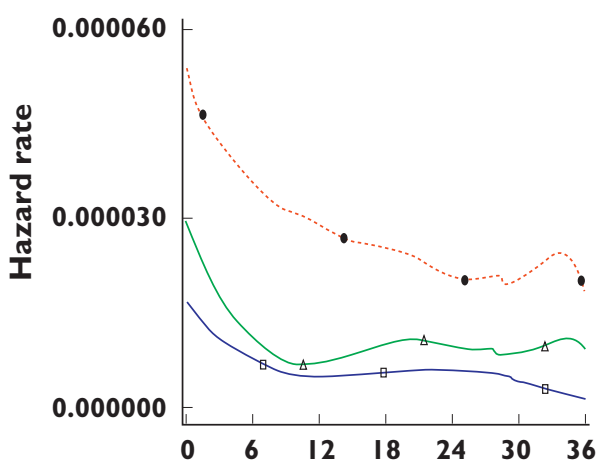

Overdose

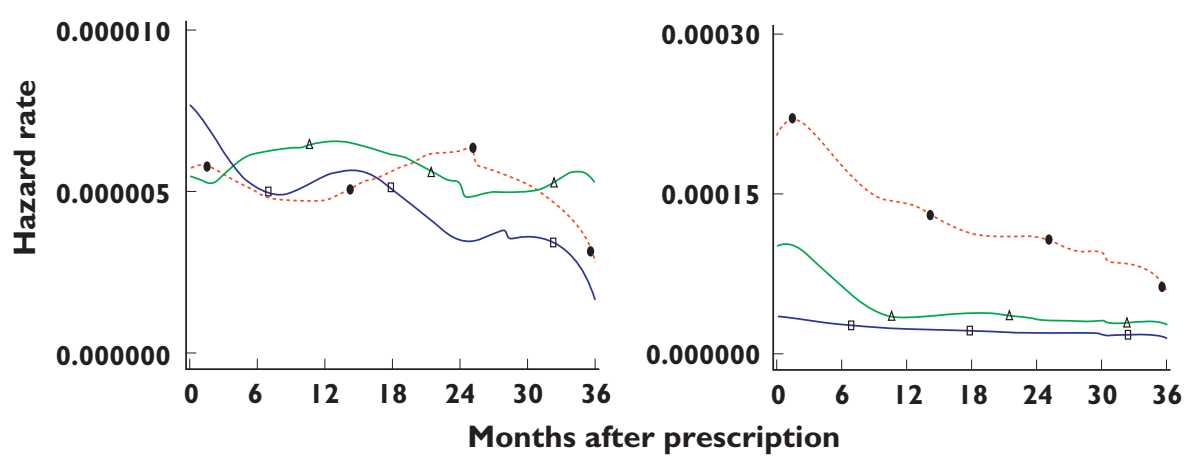

Myocardial infarction

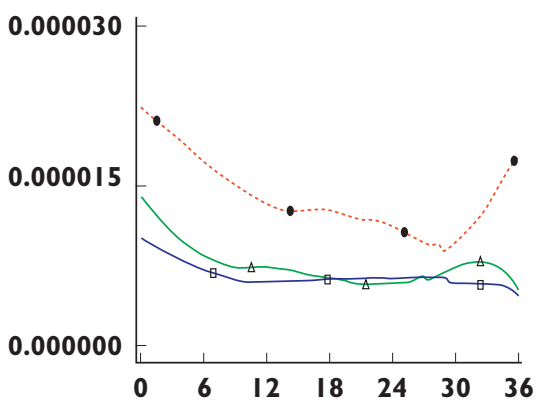

Months after prescription

Renal failure

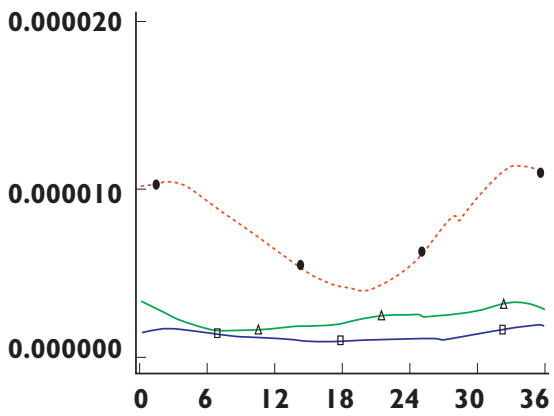

Months after prescription

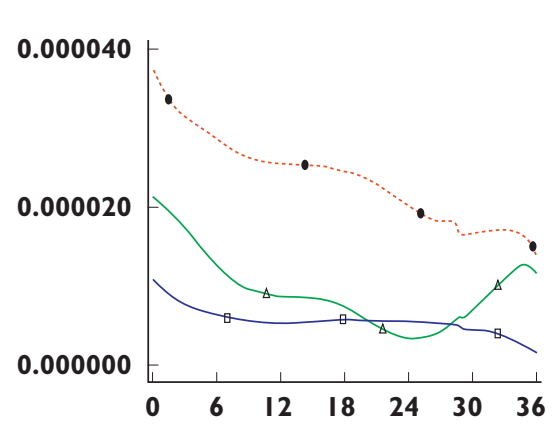

Suicidal behaviour

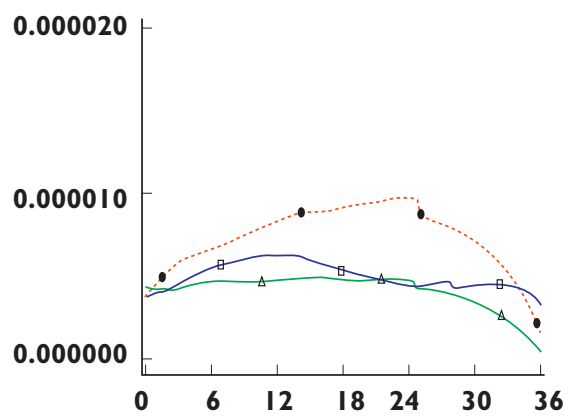

Figure 1

Pattern of the crude hazard rates for safety outcomes in the 36 months following an ibuprofen and/or paracetamol prescription. Concomitant paracetamol and ibuprofen $(\Delta)$, ibuprofen only $(\square)$, paracetamol only $(\mathbf{O})$

observed to be increased substantially around the time of the first NSAID prescription. This finding may be explained by protopathic bias, which occurs when a drug is inadvertently prescribed for an early manifestation of a disease that has not been diagnostically detected [22]. This bias has been described in other settings [7, 23]. For example, a study in the Netherlands found that recent starters of $\beta_{2}$-adrenoceptor agonists have an increased MI risk, especially patients with a history of ischaemic heart disease [23].

\section{Main limitations of the study}

Patient exposure was based on prescription information rather than actual use. In addition both paracetamol and ibuprofen are available OTC. Use of OTC medications is rarely recorded by GPs and patients prescribed ibuprofen 
or paracetamol as monotherapy might also be taking the other or another NSAID as an OTC. This may result in misclassification of past users and concomitant ibuprofen and paracetamol use, which would have resulted in an underestimate of any treatment effect. Because there are no data in the public domain on the actual intake of OTC medications in the population that we have studied, we were not able to quantify the extent of the misclassification of exposure and the impact on the relative rates. Another limitation is that ibuprofen and paracetamol prescribed on the same date may not necessarily be used by patients at the same time. This again is likely to have underestimated any effects of concomitant ibuprofen and paracetamol use.

As expected with an observational study, information for some of the risk factors associated with the outcomes is incomplete (e.g. details on disease severity). The pattern analysis evaluated the presence of differential effects that varied between current and past exposure. A limitation of this pattern analysis, similar to standard epidemiological analyses, is bias by time-dependent confounding (i.e. differential changes in risk factors in the comparison groups over time).

\section{Overall conclusion}

There was considerable heterogeneity in the patient and exposure characteristics between groups. The RRs and hazard rate patterns were statistically similar for most safety outcomes between patients prescribed ibuprofen and paracetamol concomitantly and those prescribed ibuprofen or paracetamol alone. This suggests that concomitant use of ibuprofen and paracetamol does not increase risk of the various safety outcomes examined over use of paracetamol or ibuprofen alone.

\section{Competing interests}

This study was funded by Reckitt Benckiser. The GPRD is owned by the UK Department of Health and operates within the MHRA. The GPRD is funded by the MHRA, Medical Research Council, various universities, contract research organizations and pharmaceutical companies. The Department of Pharmacoepidemiology \& Pharmacotherapy, Utrecht Institute for Pharmaceutical Sciences has received unrestricted funding for pharmacoepidemiological research from GlaxoSmithKline, Novo Nordisk, the private-public-funded Top Institute Pharma (www.tipharma.nl) includes co-funding from universities, government, and industry, the Dutch Medicines Evaluation Board, and the Dutch Ministry of Health. The authors also acknowledge the editorial assistance provided by Elements Communications Ltd, Westerham, Kent, UK. There are no other competing interests to declare.

\section{REFERENCES}

1 Hernandez-Diaz S, Garcia-Rodriguez LA. Epidemiologic assessment of the safety of conventional nonsteroidal anti-inflammatory drugs. Am J Med 2001; 110: 20S-27S.

2 Parkinson J, Davis S, van Staa TP. The General Practice Research (GPRD) Database: now and the future. In: Pharmacovigilance, ed. Mann R. Chichester: John Wiley \& Sons Ltd, 2007; 341-8.

3 Walley T, Mantgani A. The UK General Practice Research Database. Lancet 1997; 350: 1097-9.

4 Martinez C, Rietbrock S, Wise L, Ashby D, Chick J, Moseley J, Evans S, Gunnell D. Antidepressant treatment and the risk of fatal and non-fatal self harm in first episode depression: nested case-control study. BMJ 2005; 330: 389.

5 Ramlau-Hansen H. Smoothing counting process intensities by means of kernel functions. Ann Statist 1983; 11:453-66.

6 Zhang B, de Vries F, Setakis E, van Staa TP. The pattern of myocardial infarction in patients taking asthma medication: a study with the General Practice Research Database. J Hypertens 2009; 27: 1485-92.

7 De Vries F, Setakis E, Zhang B, van Staa TP. Long-acting beta2-agonists in adult asthma and the pattern of risk of death and severe asthma outcomes: a study with the General Practice Research Database. Eur Respir J 2010; doi:10.1183/09031936.00124209 [published online 29 March 2010].

8 Lipworth L, Friis S, Mellemkjaer L, Signorello LB, Johnsen SP, Nielsen GL, McLaughlin JK, Blot WJ, Olsen JH. A population-based cohort study of mortality among adults prescribed paracetamol in Denmark. J Clin Epidemiol 2003; 56: 796-801.

9 Lipworth L, Friis S, Blot WJ, McLaughlin JK, Mellemkjaer L, Johnsen SP, Nørgaard B, Olsen JH. A population-based cohort study of mortality among users of ibuprofen in Denmark. Am J Ther 2004; 11: 156-63.

10 García Rodríguez LA, Hernández-Díaz S. Relative risk of upper gastrointestinal complications among users of acetaminophen and nonsteroidal anti-inflammatory drugs. Epidemiology 2001; 12:570-6.

11 Hippisley-Cox J, Coupland C, Logan R. Risk of adverse gastrointestinal outcomes in patients taking cyclo-oxygenase- 2 inhibitors or conventional non-steroidal anti-inflammatory drugs: population based nested case-control analysis. BMJ 2005; 331: 1310-6.

12 Setakis E, Leufkens HG, van Staa TP. Changes in the characteristics of patients prescribed selective cyclooxygenase 2 inhibitors after the 2004 withdrawal of rofecoxib. Arthritis Rheum 2008; 59: 1105-11.

13 Rahme E, Barkun A, Nedjar H, Gaugris S, Watson D. Hospitalizations for upper and lower Gl events associated with traditional NSAIDs and acetaminophen among the elderly in Quebec, Canada. Am J Gastroenterol 2008; 103: 872-82.

14 Lewis JD, Kimmel SE, Localio AR, Metz DC, Farrar JT, Nessel L, Brensinger C, McGibney K, Strom BL. Risk of serious upper 
gastrointestinal toxicity with over-the-counter nonaspirin nonsteroidal anti-inflammatory drugs. Gastroenterology 2005; 129: 1865-74.

15 Chan AT, Manson JE, Albert CM, Chae CU, Rexrode KM, Curhan GC, Rimm EB, Willett WC, Fuchs CS. Nonsteroidal antiinflammatory drugs, acetaminophen, and the risk of cardiovascular events. Circulation 2006; 113: 1578-87.

16 Bresalier RS, Sandler RS, Quan H, Bolognese JA, Oxenius B, Horgan K, Lines C, Riddell R, Morton D, Lanas A, Konstam MA, Baron JA, Adenomatous Polyp Prevention on Vioxx (APPROVe) Trial Investigators. Cardiovascular events associated with rofecoxib in a colorectal adenoma chemoprevention trial. N Engl J Med 2005; 352: 1092-102.

17 Solomon SD, McMurray JJV, Pfeffer MA, Wittes J, Fowler R, Finn P, Anderson WF, Zauber A, Hawk E, Bertagnolli A. Cardiovascular risk associated with celecoxib in a clinical trial for colorectal adenoma prevention. N Engl J Med 2005; 352: 1071-80.

18 Nussmeier NA, Whelton AA, Brown MT, Langford RM, Hoeft A, Parlow JL, Boyce SW, Verburg KM. Complications of the COX-2 inhibitors parecoxib and valdecoxib after cardiac surgery. N Engl J Med 2005; 352: 1081-91.

19 Psaty BM, Furberg CD. COX-2 inhibitors - lessons in drug safety. N Engl J Med 2005; 352: 1133-5.

20 McGettigan P, Henry D. Cardiovascular risk and inhibition of cyclooxygenase: a systematic review of the observational studies of selective and nonselective inhibitors of cyclooxygenase 2. JAMA 2006; 296: 1633-44.

21 van Staa TP, Rietbrock S, Setakis E, Leufkens HG. Does the varied use of NSAIDs explain the differences in the risk of myocardial infarction? J Intern Med 2008; 264: 481-92.

22 Squire I. Shortness of breath, prescription of bronchodilators and the risk of myocardial infarction. J Hyperten 2009; 27: 1358-9.

23 de Vries F, Pouwels S, Bracke M, Lammers JWJ, Klungel OH, Leufkens HGM, Bracke $M$, van Staa TP. Use of beta- 2 agonists and risk of acute myocardial infarction in patients with hypertension. Br J Clin Pharmacol 2008; 65: 580-6. 